The method here described is based on the suggestions made by Mr. Frederick King. These suggestions have led to the evaluation of $R(x)$ as a starting point of the subsequent discussion.

New York City

\title{
ALL INTEGERS EXCEPT 23 AND 239 ARE SUMS OF EIGHT CUBES
}

\section{E. DICKSON}

Summary. In 1770 Waring stated that every positive integer is a sum of nine integral nonnegative cubes. The first proof is due to Wieferich.* I shall prove the following new result.

THEOREM. Every positive integer other than 23 and 239 is a sum of eight integral nonnegative cubes.

Five lemmas are required.

LEMMA 1. Every integer greater than or equal to $233^{6} D$ is a sum of eight cubes if $D=14.0029682$, or more generally if $D=d$, where $\dagger$

$$
d>14+\left(\frac{24}{167}\right)^{3}, \quad d \leqq 14.1 .
$$

The algebraic part of Wieferich's proof holds for all integers exceeding $2 \frac{1}{4}$ billion. The fact that all smaller integers are sums of nine cubes was proved by use of Table I. To prove my theorem, I shall need also the new Tables II and III.

Table I gives, for each positive integer $N \leqq 40,000$, the least number $m$ such that $N$ is a sum of $m$ cubes.

It was computed by R. D. von Sterneck $\ddagger$ by adding all cubes to

* His errors are avoided in the much simpler proof by the writer, Transactions of this Society, vol. 30 (1928), pp. 1-18. On page 16 is proved a generalization of Landau's result that all sufficiently large numbers are sums of eight cubes.

$\dagger$ The proof is essentially like that given for $d=14.1$ by W. S. Baer, Beitrage zum Waringschen Problem, Dissertation, Göttingen, 1913.

$\ddagger$ Sitzungsberichte der Akademie der Wissenschaften, Vienna, IIa, vol. 112 (1903), pp. 1627-1666. 
each sum of $j$, but not fewer, cubes for $j=1,2, \cdots$. Any error in the $j$ th step would introduce an increasingly large number of errors in the later steps. For this reason, my assistant, Miss Evelyn Garbe, computed in four weeks the table* for $12,000<N \leqq 40,000$ by my improved method described later. The only error found is

$$
32,822=3^{3}+3^{3}+32^{3},
$$

whose $m$ was given to be 4 by von Sterneck. The latter stated that his table agrees completely with the table for $N \leqq 12,000$ computed by Dahse and published by Jacobi. $\dagger$

Table II is a continuation to 123,000 of Table I.

Tables I and II imply the following fact.

LEMma 2. All integers from 8,043 to 123,000 are sums of six cubes.

Table III is a list of all integers from 123,000 to 560,000 which are sums $C_{4}$ of four or fewer positive cubes.

The entries not found twice were computed independently, and likewise for the auxiliary list of $C_{3}$. Allowing for permutations of the cubes in a sum of them, when making a table of sums of $r+1$ cubes, we need add a fixed cube $c^{3}$ only to a sum of $r$ cubes which is not less than $r c^{3}$. When finding the $C_{4}>400,000$ we saved much time by adding to the $C_{3}$ no cubes greater than 64,000 , but found separately the $C_{4}$ which are sums of cubes all greater than 64,000 (without new computation for necessary $C_{2}$ and $C_{3}$ ).

The entries in Table III which are greater than or equal to $C=400,000$ and less than $D=401,000$, for example, appear on a double sheet. To deduce rapidly a provisional list $\mathrm{L}^{\prime}$ of $C_{5}$ between $C$ and $D$, we compiled the entries of that double sheet, the same entries increased by unity, and the entries of the double sheet for the interval from $C-(10 k)^{3}$ to $D-(10 k)^{3}$ for $k=1,2, \cdots$, so that such an entry increased by $(10 k)^{3}$ is a $C_{5}$. From each number missing from the list $L^{\prime}$ we subtracted successive cubes to see if the difference is in Table III. In this manner we obtain the following list $\mathrm{L}$ of all integers between $A$ and $B$ which are not sums of five or fewer cubes. Note that $125,564,131,423$, and 259,682 are the only entries $E$ such that $E-1$ is also an entry. Since $E-8$ and $N-1$, when $N \neq E$, are not in $\mathrm{L}$, we conclude that every number in $\mathrm{L}$ is a $C_{6}$.

* But without checking that no number marked $m=6$ is a sum of 5 cubes (it certainly is not a sum of four or fewer cubes). Such a decision is unnecessary for the applications in this paper.

$\dagger$ Journal für die reine und angewandte Mathematik, vol. 42 (1851), pp. 41-69; Werke, vol. 6, pp. 322-354. 
LEMMA 3. All integers from 123,000 to 560,000 are sums of six cubes.

List* L of All Integers Between 123,000 ANd 560,000

Which Are Sums of Six, but Not Fewer, Cubes

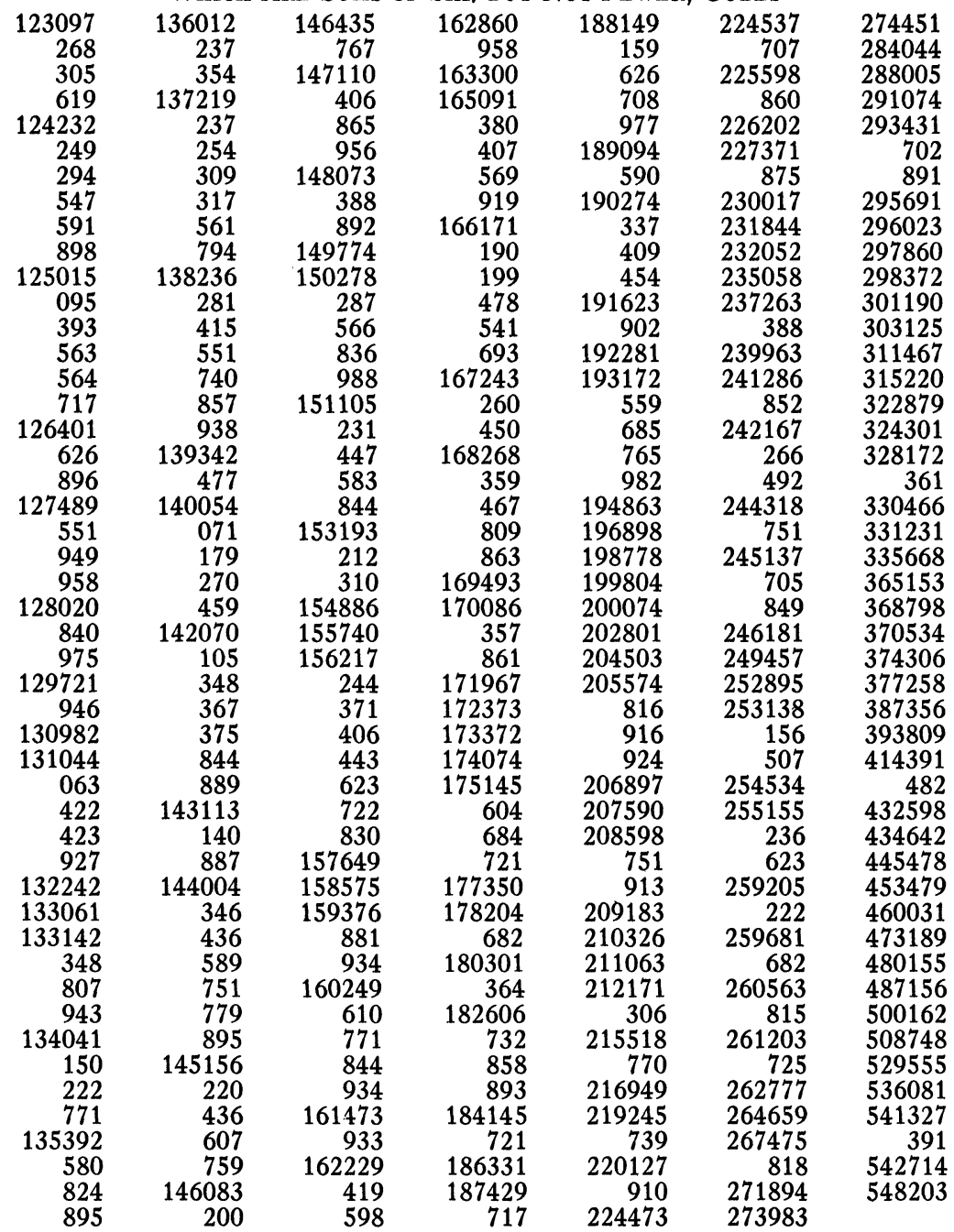

* This short table is remarkable in that it gives the essential data about sums of cubes for a range of about 440,000 numbers. By its use alone it is shown that all integers from 123,000 to $21,000,000$ are sums of six cubes.

To extend Lemma 3 beyond $B=560,000$, note that when $44^{3}$ is added to the numbers from 474,816 to $B$, we get the numbers from $B$ to $F=645,184$, which will be proved to be $C_{6}$. This is evidently true when $44^{3}$ is added to a number not in $\mathrm{L}$, since it is a $C_{5}$. Hence we 
need only add $44^{3}$ to the last ten numbers $N$ of $\mathrm{L}$. Theoretically we subtract $45^{3}$ from each sum. Actually we subtract $45^{3}-44^{3}=5,941$ from those ten numbers $N$, and note that the differences are not in $\mathrm{L}$ and hence are $C_{5}$. Thus $N+44^{3}=45^{3}+C_{5}$ is a $C_{6}$.

Next we added $69^{3}$ to the numbers from $G=316,675$ to $B$ to get the numbers from $F$ to 888,509 , which are all $C_{6}$. In fact, if we subtract $70^{3}-69^{3}=14,491$ from the last 32 numbers of $\mathrm{L}$ (that is, those greater than $G$ ), no difference is in $\mathrm{L}$.

After 102 more such simple steps we find, by use of List $\mathrm{L}$ alone, that all integers from $B$ to $B+273^{3}=20,906,417$ are sums of six cubes.

For the next step, $335,668+274^{3}-275^{3}=109,617$ is below the limit for List $\mathrm{L}$, but is a $C_{5}$ by Table II.

The first case of new type is

$$
278^{3}+500,162=279^{3}+267,475=280^{3}+33,114,
$$

where 267,475 is in $\mathrm{L}$; but 33,114 is a $C_{5}$. The next such case is

$$
284^{3}+393,809=285^{3}+150,988=283^{3}+H, \quad H=634,926 .
$$

But $H-43^{3}=555,419$ is a $C_{4}$ by Table III. Continuing, and combining the result with Lemmas 2 and 3, we obtain the next lemma.

LEMMA 4. All integers from 8,043 to $J=41,623,625$ are sums of six cubes.

LEMmA 5. Given a positive integer $S$ and $a$ number $B$ satisfying $0 \leqq B \leqq S$, we can find* an integer $i \geqq 0$ such that

$$
B \leqq S-i^{3}<B+3 S^{2 / 3} \text {. }
$$

Take $B=8,043$ and choose $S$ so that $S \geqq 8,043, B+3 S^{2 / 3} \leqq J$, which holds if $\log S \leqq 10.7132020$. By Lemma $4, S-i^{3}$ is a $C_{6}$, whence $S$ is a $C_{7}$.

By Table I, 454 is the last integer less than 8,043 which requires eight cubes. Hence all integers from 455 to the preceding $S$ are $C_{7}$. In Lemma 5 with $S$ replaced by $s$, take $B=455$ and $B+3 s^{2 / 3}=S$. Then $s-i^{3}$ is a $C_{7}$, so that $S$ is a $C_{8}$, if $\log S=15.3541210, S=22.60065$ $\times 10^{14}$. Since $S>233^{6} \mathrm{D}$, the present result together with Lemma 1 shows that every integer not less than 455 is a $C_{8}$. Below 455 , Table I shows that 23 and 239 are the only integers which require nine cubes. This proves our theorem. The manuscript of Tables II and III has been given to the library of the University of Chicago.

\section{The University of Chicago}

\footnotetext{
* Transactions of this Society, vol. 30 (1928), p. 4, case $t=1$.
} 